\title{
Setup for the measurement of the ${ }^{235} U(n, f)$ cross section relative to $n-p$ scat- tering up to $1 \mathrm{GeV}$
}

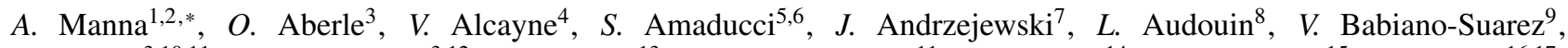
M. Bacak $^{3,10,11}$, M. Barbagallo ${ }^{3,12}$, S. Bennett ${ }^{13}$, E. Berthoumieux ${ }^{11}$, D. Bosnar ${ }^{14}$, A. S. Brown ${ }^{15}$, M. Busso ${ }^{16,17}$, M. Caamaño ${ }^{18}, L$. Caballero ${ }^{9}, M$. Calviani ${ }^{3}, F$. Calviño ${ }^{19}, D$. Cano-Ott ${ }^{4}, A$. Casanovas ${ }^{19}, F$. Cerutti $^{3}, E$. Chiaveri $^{13,20,3}$, N. Colonna ${ }^{12}$, G. P. Cortés ${ }^{19}, M$. A. Cortés-Giraldo ${ }^{20}, L$. Cosentino $^{5}, S$. Cristallo ${ }^{16,21}, L$. A. Damone ${ }^{12,22}, P$. J. Davies $^{13}$, M. Diakaki ${ }^{23}, M$. Dietz $^{24}, C$. Domingo-Pardo ${ }^{9}, R$. Dressler ${ }^{25}, Q$. Ducasse ${ }^{26}, E$. Dupont ${ }^{11}, I$. Durán ${ }^{18}, Z$. Eleme ${ }^{27}$, $B$. Fernández-Domíngez ${ }^{18}, A$. Ferrari ${ }^{3}, I$. Ferro-Gonçalves ${ }^{28}, P$. Finocchiaro ${ }^{5}, V$. Furman ${ }^{29}, R$. Garg ${ }^{24}, A$. Gawlik ${ }^{7}$, $S$. Gilardoni ${ }^{3}, K$. Göbel $^{30}, E$. González-Romero ${ }^{4}, C$. Guerrero ${ }^{20}, F$. Gunsing ${ }^{11}, S$. Heinitz ${ }^{25}, J$. Heyse ${ }^{31}, D$. G. Jenkins ${ }^{15}$, E. Jericha ${ }^{10}, U$. Jiri ${ }^{25}, A$. Junghans $^{32}, Y . \mathrm{Kadi}^{3}, F$. Käppeler ${ }^{33}, A . \mathrm{Kimura}^{34}, I$. Knapová ${ }^{35}, M$. Kokkoris ${ }^{23}, Y$. Kopatch ${ }^{29}$, $M$. Krtička ${ }^{35}, D$. Kurtulgil $^{30}, I$. Ladarescu ${ }^{9}, C$. Lederer-Woods ${ }^{24}, J$. Lerendegui-Marco ${ }^{20}, S .-J$. Lonsdale $^{24}, D$. Macina $^{3}$,

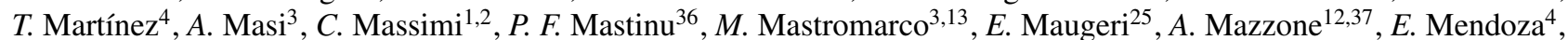
A. Mengoni ${ }^{38,1}$, V. Michalopoulou ${ }^{3,23}$, P. M. Milazzo $^{39}$, M. A. Millán-Callado ${ }^{20}$, F. Mingrone ${ }^{3}$, J. Moreno-Soto ${ }^{11}$,

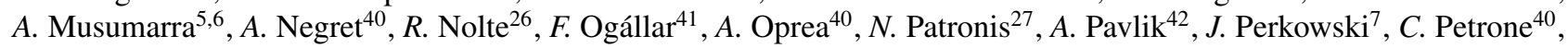
L. Piersanti ${ }^{16,21}, E$. Pirovano ${ }^{26}, I$. Porras $^{41}, J$. Praena ${ }^{41}, J . M$. Quesada ${ }^{20}, D$. Ramos Doval ${ }^{8}, R$. Reifarth ${ }^{30}, D$. Rochman $^{25}$, C. Rubbia ${ }^{3}$, M. Sabaté-Gilarte ${ }^{20,3}, A$. Saxena ${ }^{43}$, P. Schillebeeckx ${ }^{31}$, D. Schumann ${ }^{25}$, A. Sekhar ${ }^{13}$, A. G. Smith ${ }^{13}$, N. Sosnin ${ }^{13}, P$. Sprung ${ }^{25}, A$. Stamatopoulos ${ }^{23}, G$. Tagliente ${ }^{12}, J$. L. Tain ${ }^{9}$, A. E. Tarifeño-Saldivia ${ }^{19}, L$. Tassan-Got ${ }^{3,23,8}$, B. Thomas $^{30}, P$. Torres-Sánchez ${ }^{41}, A$. Tsinganis ${ }^{3}, S$. Urlass ${ }^{3,32}, S$. Valenta ${ }^{35}, G$. Vannini ${ }^{1,2}, V . V_{\text {Variale }}^{12}, P . V^{28}{ }^{28}$, $A$. Ventura ${ }^{1}, D$. Vescovi ${ }^{16,44}, V$. Vlachoudis ${ }^{3}, R$. Vlastou ${ }^{23}, A$. Wallner ${ }^{45}, P$. J. Woods ${ }^{24}, T$. J. Wright ${ }^{13}$, and $P$. Žugec ${ }^{14}$

${ }^{1}$ Istituto Nazionale di Fisica Nucleare, Sezione di Bologna, Italy

${ }^{2}$ Dipartimento di Fisica e Astronomia, Università di Bologna, Italy

${ }^{3}$ European Organization for Nuclear Research (CERN), Switzerland

${ }^{4}$ Centro de Investigaciones Energéticas Medioambientales y Tecnológicas (CIEMAT),Spain

${ }^{5}$ INFN Laboratori Nazionali del Sud, Catania, Italy

${ }^{6}$ Dipartimento di Fisica e Astronomia, Università di Catania, Italy

${ }^{7}$ University of Lodz, Poland

${ }^{8}$ IPN, CNRS-IN2P3, Univ. Paris-Sud, Université Paris-Saclay, F-91406 Orsay Cedex,France

${ }^{9}$ Instituto de Física Corpuscular, CSIC - Universidad de Valencia, Spain

${ }^{10}$ Technische Universität Wien, Austria

${ }^{11}$ CEA Saclay, Irfu, Université Paris-Saclay, Gif-sur-Yvette, France

${ }^{12}$ Istituto Nazionale di Fisica Nucleare, Bari, Italy

${ }^{13}$ University of Manchester, United Kingdom

${ }^{14}$ Department of Physics, Faculty of Science, University of Zagreb, Croatia

${ }^{15}$ University of York, United Kingdom

${ }^{16}$ Istituto Nazionale di Fisica Nazionale, Perugia, Italy

${ }^{17}$ Dipartimento di Fisica e Geologia, Università di Perugia, Italy

${ }^{18}$ University of Santiago de Compostela, Spain

${ }^{19}$ Universitat Politècnica de Catalunya, Spain

${ }^{20}$ Universidad de Sevilla, Spain

${ }^{21}$ Istituto Nazionale di Astrofisica - Osservatorio Astronomico d'Abruzzo, Italy

${ }^{22}$ Dipartimento di Fisica, Università degli Studi di Bari, Italy

${ }^{23}$ National Technical University of Athens, Greece

${ }^{24}$ School of Physics and Astronomy, University of Edinburgh, United Kingdom

${ }^{25}$ Paul Scherrer Institut (PSI), Villigen, Switzerland

${ }^{26}$ Physikalisch-Technische Bundesanstalt (PTB), Bundesallee 100, 38116 Braunschweig, Germany

${ }^{27}$ University of Ioannina, Greece

${ }^{28}$ Instituto Superior Técnico, Lisbon, Portugal

${ }^{29}$ Joint Institute for Nuclear Research (JINR), Dubna, Russia

${ }^{30}$ Goethe University Frankfurt, Germany

${ }^{31}$ European Commission, Joint Research Centre, Geel, Retieseweg 111, B-2440 Geel,Belgium

${ }^{32}$ Helmholtz-Zentrum Dresden-Rossendorf, Germany

${ }^{33}$ Karlsruhe Institute of Technology, Campus North, IKP, 76021 Karlsruhe, Germany

${ }^{34}$ Japan Atomic Energy Agency (JAEA), Tokai-mura, Japan 
${ }^{35}$ Charles University, Prague, Czech Republic

${ }^{36}$ Istituto Nazionale di Fisica Nucleare, Sezione di Legnaro, Italy

${ }^{37}$ Consiglio Nazionale delle Ricerche, Bari, Italy

${ }^{38}$ Agenzia nazionale per le nuove tecnologie, l'energia e lo sviluppo economico sostenibile (ENEA), Bologna, Italy

${ }^{39}$ Istituto Nazionale di Fisica Nazionale, Trieste, Italy

${ }^{40}$ Horia Hulubei National Institute of Physics and Nuclear Engineering (IFIN-HH),Bucharest

${ }^{41}$ University of Granada, Spain

${ }^{42}$ University of Vienna, Faculty of Physics, Vienna, Austria

${ }^{43}$ Bhabha Atomic Research Centre (BARC), India

${ }^{44}$ Gran Sasso Science Institute (GSSI), L'Aquila, Italy

${ }^{45}$ Australian National University, Canberra, Australia

\begin{abstract}
The neutron induced fission of ${ }^{235} \mathrm{U}$ is extensively used as a reference for neutron fluence measurements in various applications, ranging from the investigation of the biological effectiveness of high energy neutrons, to the measurement of high energy neutron cross sections of relevance for accelerator driven nuclear systems. Despite its widespread use, no data exist on neutron induced fission of ${ }^{235} \mathrm{U}$ above $200 \mathrm{MeV}$. The neutron facility n_TOF offers the possibility to improve the situation. The measurement of ${ }^{235} \mathrm{U}(\mathrm{n}, \mathrm{f})$ relative to the differential n-p scattering cross-section, was carried out in September 2018 with the aim of providing accurate and precise cross section data in the energy range from $10 \mathrm{MeV}$ up to $1 \mathrm{GeV}$. In such measurements, Recoil Proton Telescopes (RPTs) are used to measure the neutron flux while the fission events are detected and counted with dedicated detectors. In this paper the measurement campaign and the experimental set-up are illustrated.
\end{abstract}

\section{Introduction}

Neutron cross section standards are fundamental ingredients for both measurements and evaluations of neutroninduced reaction cross sections. However, no cross section standard exists for neutron energies above $200 \mathrm{MeV}$. This led the International Atomic Energy Agency to issue a request for a new absolute measurement of neutron induced fission cross sections, relative to n-p scattering, to establish a fission cross section standard above $200 \mathrm{MeV}$ [1]. An effective choice for the reaction to be studied is the ${ }^{235} \mathrm{U}(\mathrm{n}, \mathrm{f})$ reaction, already one of the most important standard cross section at thermal neutron energy and between $0.15 \mathrm{MeV}$ and $200 \mathrm{MeV}$ [2]. Despite its importance in fundamental nuclear physics and its widespread use as reference for neutron fluence measurements, only two data sets are available in the energy range between 20 and $200 \mathrm{MeV}$ and two absolute experimental measurements have been performed in the high energy region, above $200 \mathrm{MeV}[3,4]$ (figure 1). Moreover the fission process of Uranium at high excitation energy is an important topic as it is related to fundamental quantities of excited nuclear matter, like the viscosity and transient effects [10, 11].

Thanks to the intense neutron beam, with a wide energy spectrum ranging from thermal to $1 \mathrm{GeV}$, the n_TOF facility at CERN can answer to the request of the IAEA for new measurements. A dedicated measurement campaign was carried out with the aim of providing accurate and precise cross section data of the ${ }^{235} \mathrm{U}(\mathrm{n}, \mathrm{f})$ reaction in the high energy region.

\section{Experimental set-up}

Neutrons at n_TOF (see [13] for an extensive description) are produced by spallation reaction: bunches of protons of $20 \mathrm{GeV} / \mathrm{c}$ momentum are shot every few seconds by the

*e-mail: alice.manna@bo.infn.it

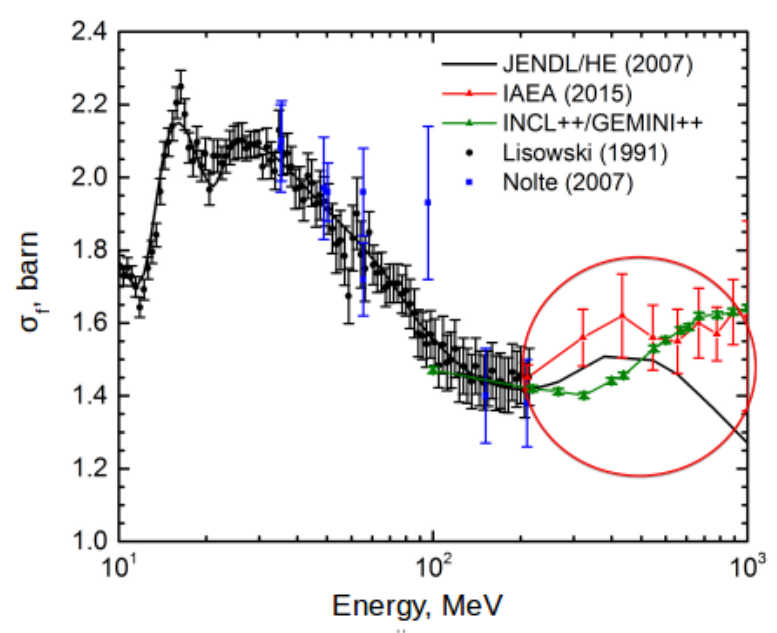

Figure 1. The ${ }^{235} \mathrm{U}(\mathrm{n}, \mathrm{f})$ cross section from the JENDL/HE [5] and IAEA [1] evalutations, the experimental data [6,7] measured relative to the n-p cross section and a new theoretical calculation [8] based on the intranuclear cascade model INCL++ [9] coupled to the deexcitation model GEMINI++ [12].

CERN Proton Synchrotron on a massive lead target; about 300 neutrons are produced per incident proton. The facility features two beam lines with a white neutrons spectrum, and two experimental areas at the nominal distance of $185 \mathrm{~m}$ for EAR-1 [14] and $20 \mathrm{~m}$ for EAR-2 [15] from the neutron-producing target.

The measurement of the ${ }^{235} \mathrm{U}(\mathrm{n}, \mathrm{f})$ cross section was performed in September 2018 in the first experimental area, where the neutron spectrum extends up to $1 \mathrm{GeV}$, and also to profit from the good energy resolution in the high energy region, thanks to the longer flight path.

The experimental setup included detectors to count the fission events from the Uranium target and, at the same time, 
detectors to measure the neutron fluence relative to the neutron-proton elastic scattering reaction, the primary reference for neutron cross sections. Fission events were detected using an ionisation chamber from the PhysikalischTechnische Bundesanstalt [16] and a reaction chamber based on Parallel Plate Avalanche Counters (PPACs) developed at Institut de Physique Nucléaire d'Orsay [17, 18]. Three recoil proton telescopes (RPTs), developed by the National Institute of Nuclear Physics (INFN, Italy) and the Physikalisch-Technische Bundesanstalt (PTB, Germany), are used to measure the neutron fluence. Figure 2 shows, the scheme of the experimental set up: the two fission chambers with a total of ten fissile deposits placed in the neutron beam, downstream two Polyethylene $\left(\mathrm{C}_{2} \mathrm{H}_{4}\right)$ targets and the three telescopes placed at a small angle with respect to the neutron beam.
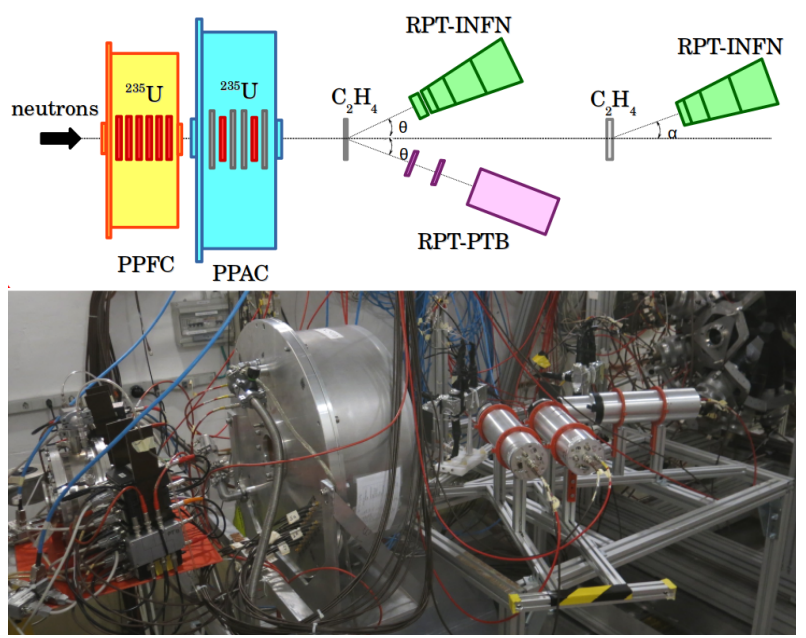

Figure 2. Layout of the ${ }^{235} \mathrm{U}(\mathrm{n}, \mathrm{f})$ relative to ${ }^{1} \mathrm{H}(\mathrm{n}, \mathrm{n}){ }^{1} \mathrm{H}$ cross section measurement set-up carried out in EAR-1 at $n \_$TOF. Fission reactions are measured with a fission chamber and a set of Parallel Plate Avalanche Counters. Three telescopes pointing at two different $\mathrm{C}_{2} \mathrm{H}_{4}$ radiators were used to measure the neutron fluence.

\subsection{Fission reaction detection}

The Parallel Plate Avalanche Counters consist of 3 electrodes, a central anode surronded by two cathodes; the 3 $\mathrm{mm}$ gaps between electrodes are filled with forced flow of $\mathrm{C}_{3} \mathrm{~F}_{8}$ maintained at low-pressure $(4 \mathrm{mbar})$. The electrodes are made of $1.7 \mu \mathrm{m}$ thick mylar foils, coated with aluminium or gold to make them conductive. The coating in the cathodes is divided into $2 \mathrm{~mm}$ wide strips to allow the localisation of the avalanche by using a delay line; the time difference between delay line outputs provides a onedimensional position. By combining the signals from the two orthogonal cathodes strips, the fission fragment trajectory can be reconstructed. The fission reaction chamber includes 3 PPACs with two ${ }^{235} \mathrm{U}$ samples in between. Each sample consisted in a $8 \mathrm{~cm}$ diameter, $300 \mu \mathrm{g} \cdot \mathrm{cm}^{-2}$ thick layer of ${ }^{235} \mathrm{U}$ (purity of $92.7 \%$ ) that was placed on a $600 \mu \mathrm{g} \cdot \mathrm{cm}^{-2}$ aluminum backing by electrodeposition.
The fission events are identified by coincident anode signals of two consecutive PPACs. This feature, combined with the fast timing properties of the device ( 9 ns FWHM), guarantees a very high signal-to-background ratio. In addition, since these detectors are quite insensitive to the $\gamma$ flash produced by high energy reactions at the spallation target, it is possbile to recognize the events produced by $1 \mathrm{GeV}$ neutrons. However, the PPACs can detect only fission fragments emitted into a forward cone with an opening angle of about $60^{\circ}$ with a detection efficiency that is not easily evaluated.

The second system used to measure the fission reaction is an ionization chamber (PPFC) which contains a parallel plate electrode assembly with a thickness of $100 \mu \mathrm{m}$ each. The chamber is equipped with ${ }^{235} \mathrm{U}$ (purity of 99.93\%) deposits of $42 \mathrm{~mm}$ diameter and about $300 \mu \mathrm{g} \cdot \mathrm{cm}^{-2}$ areal density on both sides of four aluminum electrodes. In total 32.660(3) $\mathrm{mg}$ of ${ }^{235} \mathrm{U}$ are used. In addition, to study the background reactions due to the aluminium, there are two targets with only the backing. The chamber is operated at atmospheric pressure with a continuous gas flow of $90 \%$ argon and $10 \%$ methane mixture.

The detector is very well characterized in terms of detection uniformity of the fissile deposits and of fragment detection efficiency (about 95\%). The use of the PPFC is however limited to up to a few hundreds $\mathrm{MeV}$ of neutron energy because of the background produced by neutroninduced reactions on the aluminium foils. It is perfectly suited for the measurement of the ${ }^{235} \mathrm{U}(\mathrm{n}, \mathrm{f})$ cross section in the energy range from thermal to ca. $150 \mathrm{MeV}$; therefore, it is used to study and calibrate the PPAC fragmentdetection efficiency in the energy range below $100 \mathrm{MeV}$.

\subsection{Flux measurement set-up}

The number of neutrons impinging on the ${ }^{235} \mathrm{U}$ samples is measured simultaneously to fission by detecting recoil protons emitted from the two polyethylene targets placed downstream of the fission chambers (figure 2). Due to the presence of carbon in the samples, it is necessary to use a telescope to discriminate the protons from other charged particles exploiting the $\Delta \mathrm{E}-\mathrm{E}$ method. The energy deposited in the different layers of the telescope is different for different particles, due to the different stopping power. In non-relativistic approximation, the product between the energy loss in the transmission detectors $(\Delta \mathrm{E})$ and the remaining kinetic energy (E) in the stop detector is proportional to the charge $(\mathrm{z})$ and the mass $(\mathrm{M})$ of the interacting particle. Graphing $\Delta \mathrm{E}$ against $\mathrm{E}$ yield a family of hyperbolas corresponding to the different values of $z^{2} \mathrm{M}$.

Two RPTs were built with the same design, the third follows a different concept.

The two detectors, indicated in figure 2 as RPT-INFN (shown in detail in figure 3 ) have a compact design. The two telescopes consist of a trapezoidal structure of four independent BC408 plastic scintillators with increasing thickness: $0.5,3.0,6.0$ and $6.0 \mathrm{~cm}$. Each scintillator is coupled to a 1" Hamamatsu R1924A Photomultiplier (PMT), except for the first scintillator, that, due to the low thickness, is coupled to two PMTs (one at each side). One 

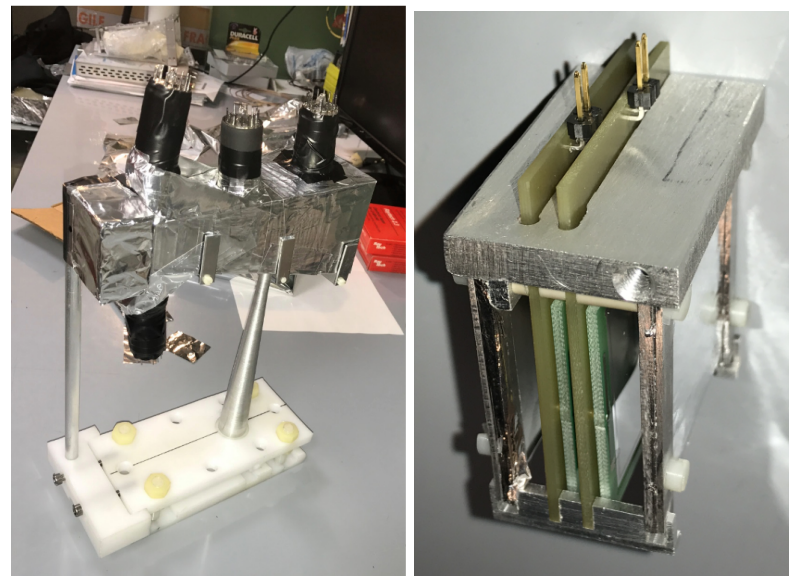

Figure 3. Left: structure of one of the two RPT-INFN: four plastic scintillators and two Silicon detectors. Right: detail of the case with the Silicon detectors inside.

of the two RPT-INFN has two silicon detectors placed before the first thin scintillator. The silicon detectors, $300 \mu \mathrm{m}$ thick inside a aluminium case (in figure 3 , right), allow to reduce the minimum detectable neutron energy from 30 $\mathrm{MeV}$ to $10 \mathrm{MeV}$.

The coincidence between the different detectors guarantees the suppression of the $\gamma$ backgroung and the events that do not come from the $\mathrm{C}_{2} \mathrm{H}_{4}$ target. Moreover, thanks to the multi-stage structure it is possible to select, for each neutron energy, the best configuration in terms of number of detectors in coincidence, to reduce the background coming from $\mathrm{n}+{ }^{12} \mathrm{C}$ reactions. The two telescopes were installed at different angles: the upstream detector was placed at $\theta=25^{\circ}$ to the neutron beam direction, and the dowstream one at $\alpha=20^{\circ}$. This allowed to increase the statistics, as well as to minimise systematic uncertainties related to the angular position.

Considering the very fast response of the scintillators, the telescope is able to identify events generated by neutrons arriving as close as $80 \mathrm{~ns}$ after the $\gamma$-flash, corresponding to an energy of $\sim 1 \mathrm{GeV}$.

The third telescope, indicated in figure 2 as RPT-PTB (shown in detail in figure 4), consists of three discrete detectors.

This triple-stage RPT has two transmission detectors that consist of a square EJ204 plastic scintillator, $45 \times 45$ $\mathrm{mm}^{2}(\Delta \mathrm{E} 1)$ and $38 \times 38 \mathrm{~mm}^{2}(\Delta \mathrm{E} 2)$ in size, respectively. The thicknesses of the detectors are optimised to the neutron energy range to be covered and vary between $0.5 \mathrm{~mm}$ and $5 \mathrm{~mm}$. Moreover the dimension of the $\Delta \mathrm{E} 2$ detector, which has the smallest transverse dimensions of all the three detectors, defines the solid angle of the RPT-PTB. The stop detector (E) is a cylindric EJ204 scintillator of $80 \mathrm{~mm}$ diameter. Also this detector thickness is optimized with respect to the energy range: $50 \mathrm{~mm}$ for neutron energy from $25 \mathrm{MeV}$ to $100 \mathrm{MeV}, 100 \mathrm{~mm}$ from $50 \mathrm{MeV}$ to $150 \mathrm{MeV}$. The $\Delta \mathrm{E}$ scintillator detectors are inserted in a rooflike housings with $0.1 \mathrm{~mm}$ aluminium walls covered with a diffuse white reflector on the inside and coupled to a XP2020Q PMTs (figure 4, right). Due to the diffuse reflec-

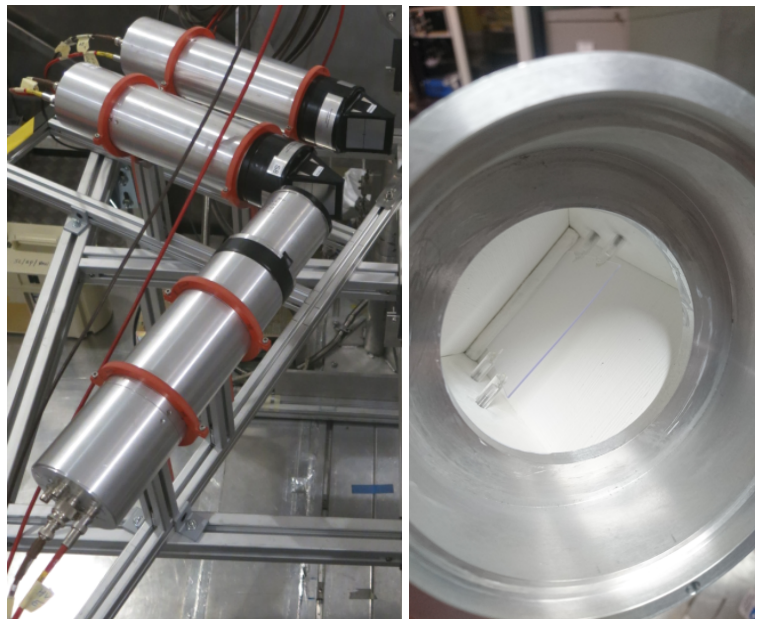

Figure 4. Left: structure of the triple-stage RPT-PTB: three plastic scintillators. Right: detail of the interior of the cover for the $\Delta \mathrm{E}$ detectors with a diffuse white reflector to reduce the inhomogeneity of the light collection.

tor the inhomogeneity of the light collection is less than $10 \%$. The envelope and the front side of the cylindrical $\mathrm{E}$ detectors are also covered with a white diffuse reflector and coupled to XP2020 PMTs.

The main design goal for this instrument is to achieve directional sensitivity for background suppression and reduced influence of angular straggling for an accurate definition of the solid angle, requiring, during the data analysis, a triple coincidence.

\section{The measurement campaign}

The ${ }^{235} \mathrm{U}(\mathrm{n}, \mathrm{f})$ measurement at $\mathrm{n} \_$TOF required a five-week beamtime in order to collect enough statistics. It started on September $17^{\text {th }}$ and ended on October $29^{\text {th }}$, for a total of $3.8 \cdot 10^{18}$ protons on target.

During the measurement campaign different $\mathrm{C}_{2} \mathrm{H}_{4}$ targets were used; their thicknesses were optimized to match the neutron-energy range of interest. In particular for the low neutron energy range (from 10 to $200 \mathrm{MeV}$ ) targets with 1 and $2 \mathrm{~mm}$ of thickness were used, whereas the corresponding carbon samples to subtract the background reaction were of 0.5 and $1 \mathrm{~mm}$ thickness. For higher neutron energies, up to $1 \mathrm{GeV}$, polyethylene and carbon samples with 5 and $2.5 \mathrm{~mm}$ thickness were used.

The RTP-PTB and the RPT-INFN with the Silicon detectors were at the same angle with respect to the neutron beam, $25^{\circ}$, and pointing at the same sample. While the third telescope, at $20^{\circ}$, was pointing always at the thicker target, to increase the statistics for the high energy part. In the table 1 the protons on target for each detector and configuration are summarized.

\section{Conclusion}

The ${ }^{235} \mathrm{U}(\mathrm{n}, \mathrm{f})$ reaction is one of the most important standard cross section both in nuclear physics and for its 
Table 1. Summary of statistics (protons on target, "pot") collected for each confguration used during the measurement campaign at $\mathrm{n}$-TOF.

\begin{tabular}{cccc}
\hline Sample & Thickness & $\begin{array}{c}\text { RPTs at 25 } \\
\text { (pot) }\end{array}$ & $\begin{array}{c}\text { RPT at 20 } \\
\text { (pot) }\end{array}$ \\
\hline \hline $\mathrm{C}_{2} \mathrm{H}_{4}$ & $1 \mathrm{~mm}$ & $7.08 \cdot 10^{17}$ & - \\
$\mathrm{C}_{2} \mathrm{H}_{4}$ & $2 \mathrm{~mm}$ & $8.41 \cdot 10^{17}$ & - \\
$\mathrm{C}_{2} \mathrm{H}_{4}$ & $5 \mathrm{~mm}$ & $7.44 \cdot 10^{17}$ & $2.06 \cdot 10^{18}$ \\
$\mathrm{C}$ & $0.5 \mathrm{~mm}$ & $3.27 \cdot 10^{17}$ & - \\
$\mathrm{C}$ & $1 \mathrm{~mm}$ & $3.86 \cdot 10^{17}$ & - \\
$\mathrm{C}$ & $2.5 \mathrm{~mm}$ & $3.14 \cdot 10^{17}$ & $1.55 \cdot 10^{18}$ \\
\hline
\end{tabular}

widespread use as reference for neutron flux measurements. Nevertheless few measurements are available in the neutron energy range between 20 and $200 \mathrm{MeV}$ and none above $200 \mathrm{MeV}$. To fulfil this lack of experimental data a campaign to measure the ${ }^{235} \mathrm{U}$ fission reaction, relative to the ${ }^{1} \mathrm{H}(\mathrm{n}, \mathrm{n})^{1} \mathrm{H}$ reaction, which is considered the primary reference in this energy region, has been carried on at the n_TOF facility at CERN in 2018. The setup was designed to measure simultaneously the fission reactions by means of two fission fragment detectors and the neutron flux using three different recoil proton telescopes. During the experimental campaign data have been acquired using different $\mathrm{C}_{2} \mathrm{H}_{4}$ and $\mathrm{C}$ targets for the evaluation of the neutron flux and systematics. The data analysis is in progress together with an intense MC simulation campaign to evaluate the detectors efficiencies using Geant-4 toolkit [19].

\section{References}

[1] B. Marcinkevicius et al., International Nuclear Data Committee, INDC(NDS) 0681, (2015)
[2] A.D. Carlson et al, Nuclear Data Sheets 110, 3215 (2009)

[3] V.I. Goldanskii et al., EXFOR data file 41212.004, J. Exptl. Theoret. Phys.(U.S.S.R.) 29, 778 (1955); Soviet Phys. JETP 2, 677 (1956)

[4] J. Rapaport et al., EXFOR data file 14036.002, LANL Report LA-11078-MS, (1987)

[5] Y. Watanabe et al., Journal of the Korean Physical Society 59, 197 (2007)

[6] A.D. Carlson et al., Proc. of the Int. Conf. on Nuclear Data for Science and Technology, Juelich (Germany) 13-17 May 1991 edited by Qaim S.M. (Springer), 518 (1992)

[7] R. Nolte et al., Nuclear Science and Engineering 156, 197 (2007)

[8] S. Lo Meo et al., Nucl. Phys. A 933, 43 (2015)

[9] A. Boudard et al., Phys. Rev. C 87, 014606 (2013)

[10] J. Taieb et al., Nucl. Phys. A 724, (2003)

[11] B. Jurado et al., Nucl. Phys. A 757, (2005)

[12] D. Mancusi et al., Phys. Rev. C 82, 044610 (2010)

[13] C. Borcea et al., Nucl. Instr. and Meth. A 513, 524 (2003)

[14] C. Guerrero et al., Eur. Phys. J A 49, 27 (2013)

[15] C. Weiss et al., NIM A 799, 90 (2015)

[16] D.B. Gayther, Metrologia 27, 221 (1990)

[17] C. Paradela et al., Phys. Rev. C 82, 034601 (2010)

[18] D. Tarrío et al., Phys. Rev. C 83, 044620 (2011)

[19] N. Terranova et al., International Conference on Nuclear Data for Science and Technology (2019), to be published 